\title{
Genomic Expression Profiling in Melanoma and the Road to Clinical Practice
}

\author{
Michael S. Sabel, MD \\ University of Michigan, Ann Arbor, MI
}

In a previous issue of the Annals of Surgical Oncology, Kangas-Dick et al. performed a retrospective review of their experience with the Decision-DX Melanoma 31-gene assay. ${ }^{1}$ Analyzing data from 361 patients, they found that while the gene expression profile (GEP) class was significantly associated with outcome in a univariate analysis, in the multivariate analysis only Breslow thickness and ulceration were associated with relapse-free survival, leading the authors to conclude that the genetic profiling did not significantly add to our ability to predict recurrence beyond traditional factors. After publication, some questions arose regarding the data and potential selection bias. Both a letter from the Editors and the reply by the senior authors, Drs. Berger and Koshenkov, appear in this issue. ${ }^{2,3}$ Valid points are raised, which highlight the hurdles that we face as we attempt to incorporate new genomic and proteomic assays into clinical practice.

These questions are not academic; we are rapidly entering a new era in oncology: tailoring treatment based on predictive and prognostic biomarkers beyond TNM staging. As technology improves and becomes more costefficient, an increasing number of genomic assays are being reported with the potential to aid in decision-making, avoiding therapies in patients unlikely to benefit and alter surveillance strategies in those more likely to recur. The potential advantages for such tools in melanoma are clear. Despite multiple revisions, the AJCC staging system has limits in its ability to stratify patients. Sentinel lymph node

(C) Society of Surgical Oncology 2021

First Received: 4 November 2021

Accepted: 4 November 2021;

Published Online: 1 December 2021

M. S. Sabel, MD

e-mail: msabel@umich.edu biopsy, while extremely useful, significantly increases the cost of care compared with wide excision alone. The present adjuvant therapies available to patients, both targeted therapies and immunotherapies, are extremely costly yet benefit a minority of patients. This is particularly poignant as we begin to debate the use of adjuvant therapy in stage II melanoma.

To tackle this, several groups have developed and introduced genomic expression profiling assays for melanoma patients. This article and the accompanying letters to the editor focus on the 31-gene assay, but several assays are being examined that may be able to stratify patients based on recurrence and survival (MelaGenix, Signatera) or by SLN status (SkylineDx, Merlin). Each hopes to have the same impact on the care of melanoma patients as the 21-gene assay (Oncotype Dx) has had on breast cancer, and it is useful to look at how this occurred. The 21-gene breast cancer assay was shown in several prospective, validation studies to be both prognostic and predictive and was ultimately incorporated into clinical guidelines for breast caner management. In all fairness, the 21-gene assay had an advantage that the melanoma assays do not; the NSABP had saved tissue from patients enrolled into randomized, clinical trials from years prior, so both tissue and long-term outcomes among patients with rigid treatment protocols were available. But the 21-gene assay also was developed and evaluated with a specific clinical question in a welldefined population, identifying patients with node-negative, hormone-sensitive breast cancer who benefit from endocrine therapy to the degree that they would not require adjuvant chemotherapy. Ultimately, the clinical benefit was demonstrated in a prospective, randomized trial (TAILORx) that confirmed this, leading to NCCN guidelines recommending Oncotype Dx as the preferred test for this population for adjuvant chemotherapy decisions. ${ }^{4}$ 
The breast cancer 21-gene assay is arguably the best example of using GEP in oncology, ushering in a new era, but the oncology literature is replete with descriptions of many forms of biomarkers that can separate groups of patients into "lower-risk" and "higher-risk" subsets. These are of biologic significance and worthy of publication, but in the absence of validation using independent datasets and studies using well-defined clinical questions and patient populations, it remains unclear whether these biomarkers have clinical utility. Imagine a biomarker that, when absent, is associated with a $20 \%$ recurrence rate, while when present is associated with a recurrence rate of $60 \%$. The biomarker is biologically significant and clearly associated with the inherent aggressiveness of the cancer. But is it clinically useful? In other words, when facing a fork in the road, does the presence or absence of this biomarker help us to choose which road to travel?

To get to that point, the first question is whether it is independent of known prognostic markers. If this biomarker strongly correlates with known prognostic factors (e.g., the biomarker is present in most node positive patients), then from a clinical perspective, it is not helpful. Additionally, our biomarker may seem impressive based on the separation of the curves, but it does not necessarily identify a group that is sufficiently low risk who could, for example, avoid adjuvant therapy or sufficiently high-risk to justify enhanced surveillance or adjuvant therapy. To this latter point, it is necessary to point out that a prognostic factor (one that identifies risk of recurrence in the absence of treatment) is not necessarily a predictive factor (one that predicts whether a patient will respond to a specific treatment). Hence, the presence of this biomarker may identify a subset of patients at higher risk of recurrence, but this does not tell you they would benefit from adjuvant therapy, because the biomarker also may correlate with resistance to available therapies.

The breast cancer 21-gene assay answered these questions by defining the "fork in the road" that they were seeking to help navigate, validating the results in a large, unselected set of patients and subsequently a prospective, randomized trial, but also had advantages that melanoma assays have not. Nonetheless, are we getting closer to determining whether the melanoma 31-gene assay is moving in the right direction? The article by Kangas-Dick et al. ${ }^{1}$ suggests that we are not, but one of the significant concerns raised is the patient population. The dataset includes cases where the test was ordered as a routine test following the SLN, but also ordered retroactively, as well as in patients who did not have a SLN biopsy. It was ordered in both patients with positive SLN as well as patients with clinical stage III disease, satellite lesions or in-transit disease. Why? Was the test used to guide adjuvant therapy decisions? The article does not discuss the use of adjuvant therapy, which clearly could have an impact on RFS. The same question arises for the patient who had a negative SLN: did the GEP class influence either adjuvant therapy or surveillance, both of which can impact RFS and DMFS.

This dilemma is not unique to the articles that failed to show a benefit. Two retrospective and three prospective studies concluded that a low-risk (class 1) score had better outcomes than high-risk (class 2). However, these studies have similarly been criticized for having varying designs, selection bias, and overlapping patient populations. ${ }^{5}$ They have performed multivariate analysis with some clinicopathologic features, but not all known features that are used for staging and decision-making. Follow-up times have been variable and often not long enough for thin melanoma, where recurrences tend to occur later. ${ }^{5,6}$

Much of the confusion and flaws in the research can be traced back to one overarching problem: the assay was made available and marketed to physicians before there were definitive studies answering the above questions, or even clearly delineating what it was to be used for. Some clinicians have used the test to determine whether patients require a SLN biopsy, even though that is not exactly what the test was developed for and the evaluation of SLN metastases was based on unadjusted analyses. ${ }^{7}$ Others state that it could be used for prognosis; however knowing a patient's prognosis is only helpful if it guides care, and there limited data that basing surveillance strategies or adjuvant therapy decisions on GEP score is warranted, let alone justifies the cost of the assay. It is being used despite the fact that current AAD and NCCN melanoma clinical practice guidelines do not endorse its use. ${ }^{8,9}$ Both groups, as well as the Melanoma Prevention Working Group, an interdisciplinary group of melanoma experts and advocates, argue that more evidence regarding the validity and clinical applicability of GEP tests is needed. ${ }^{5}$

If this GEP, and/or others, are to be incorporated into clinical protocols, cleaner evidence is needed and the assays need to be shown to be both useful and cost-effective in the framework of a specific clinical question. Unfortunately, this evidence is going to be harder to generate, and trust, if the patient populations are biased by indiscriminate use of the assay, or if the researchers are potentially biased by conflicts of interest. There are many examples of interconnectivity between industry and academia. This does not in and of itself negate conclusions, but it exemplifies the reasons why disclosures are so important and stresses the importance of independent validation with well-defined endpoints, particularly when availability of the assay surpasses evaluation of the assay. The Kangas-Dick article, post-publication questions, and author responses emphasize the limits of these studies and highlight the challenges moving forward. ${ }^{1-3}$ The potential 
benefits of GEP in melanoma are tremendous, and there is strong preliminary evidence that the 31-gene assay may ultimately fill an important clinical need. To get there, however, it is imperative to take one step back and then two steps forward, starting with retrospective analysis in representative banked specimens by independent groups, and then incorporation into prospective, clinical trial design.

DISCLOSURE MSS reports nothing to disclose.

\section{REFERENCES}

1. Kangas-Dick AW, Greenbaum A, Gall V, et al. Evaluation of a gene expression profiling assay in primary cutaneous melanoma. Ann Surg Oncol. 2021;28(8):4582-9.

2. Roh MS, Tyler DS, on behalf of the Annals of Surgical Oncology Editors. Post-publication discussion: invitation for reply. Ann Surg Oncol. 2021. https://doi.org/10.1245/s10434-021-11096-6.

3. Berger AC, Koshenkov V. Post-publication discussion: in reply. Ann Surg Oncol. 2021. https://doi.org/10.1245/s10434-021-11097-5.
4. Sparano JA, Gray RJ, Makower DF, et al. Adjuvant chemotherapy guided by a 21-gene expression assay in breast cancer. $N$ Engl $J$ Med. 2018;379(2):111-21.

5. Grossman D, Okwundu N, Bartlett EK, et al. Prognostic gene expression profiling in cutaneous melanoma: identifying the knowledge gaps and assessing the clinical benefit. Clin Rev Educ. 2020;156(9):1004-11.

6. Lo SN, Scolyer RA, Thompson JF, et al. Long-term survival of melanoma patients with thin (T1) cutaneous melanomas: a Breslow thickness cut point of $08 \mathrm{~mm}$ separates higher-risk and lower-risk tumors. Ann Surg Oncol. 2018;25(4):894-902.

7. Vetto JT, Hsueh EC, Gastman BR, et al. Guidance of sentinel lymph node biopsy decisions in patients with T1-T2 melanoma using gene expression profiling. Future Oncol. 2019;15(11):1207-17.

8. Swetter SM, Tsao H, Bichakjian CK, et al. Guidelines of care for the management of primary cutaneous melanoma. J Am Acad Dermatol. 2019;80(1):208-50.

9. National Comprehensive Cancer Network. NCCN clinical practice guidelines in oncology: cutaneous melanoma. Version 1.2021. Accessed 4 Nov 2021.

Publisher's Note Springer Nature remains neutral with regard to jurisdictional claims in published maps and institutional affiliations. 